\title{
The challenges for quantitative photoacoustic imaging
}

\author{
B. T. Cox, J. G. Laufer and P. C. Beard \\ Department of Medical Physics and Bioengineering, University College London, \\ Gower Street, London WC1E 6BT, UK \\ www.medphys.ucl.ac.uk/research/mle
}

\begin{abstract}
In recent years, some of the promised potential of biomedical photoacoustic imaging has begun to be realised. It has been used to produce good, three-dimensional, images of blood vasculature in mice and other small animals, and in human skin in vivo, to depths of several $\mathrm{mm}$, while maintaining a spatial resolution of $<100$ $\mu \mathrm{m}$. Furthermore, photoacoustic imaging depends for contrast on the optical absorption distribution of the tissue under study, so, in the same way that the measurement of optical spectra has traditionally provided a means of determining the molecular constituents of an object, there is hope that multiwavelength photoacoustic imaging will provide a way to distinguish and quantify the component molecules of optically-scattering biological tissue (which may include exogeneous, targeted, chromophores). In simple situations with only a few significant absorbers and some prior knowledge of the geometry of the arrangement, this has been shown to be possible, but significant hurdles remain before the general problem can be solved. The general problem may be stated as follows: is it possible, in general, to take a set of photoacoustic images obtained at multiple optical wavelengths, and process them in a way that results in a set of quantitatively accurate images of the concentration distributions of the constituent chromophores of the imaged tissue? If such an 'inversion' procedure not specific to any particular situation and free of restrictive suppositions - were designed, then photoacoustic imaging would offer the possibility of high resolution 'molecular' imaging of optically scattering tissue: a very powerful technique that would find uses in many areas of the life sciences and in clinical practice. This paper describes the principal challenges that must be overcome for such a general procedure to be successful.
\end{abstract}

Keywords: photoacoustic tomography, quantitative, chromphores, multiwavelength

\section{INTRODUCTION}

Consider a photoacoustic image of a region of tissue obtained using a single optical wavelength and showing a network of blood vessels. As a photoacoustic image is related to the absorption coefficient within the tissue, and recalling that a co-oximeter uses measurements of the absorption coefficient of blood at four wavelengths in order to estimate its oxygenation, it would seem a small step to obtain a map of blood oxygenation using photoacoustic imaging. More generally, if the absorption spectrum of a sample is known then it is often possible to work out the concentrations of the constituent molecules using a spectroscopic analysis. Surely multiwavelength photoacoustic imaging can, by analogy, provide maps of the concentrations of the constituent chromophores?

It is starting to be understood that spectroscopic problems like this are more challenging in photoacoustic imaging than the above analogies would suggest, and there is one principal reason for this: photoacoustic images are not images of the absorption coefficient. A set of photoacoustic images obtained at multiple wavelengths does not, therefore, give the absorption spectrum of each pixel in the image, not even to within a multiplicative constant, and naïve spectroscopic approaches are bound to fail, as has been demonstrated experimentally. ${ }^{1}$ Nevertheless, despite these difficulties, a technique that could deliver quantitatively accurate images of chromophore concentrations, or equivalently of absorption coefficient distributions, would have so many uses ${ }^{2}$ both in basic science (eg. molecular imaging of small animals) and clinically (eg. spatially resolved measurements of hemoglobin and oxygen saturation, $\mathrm{sO}_{2}$ ) that this is a goal worth working towards. This paper describes which aspects of this problem have been tackled and the challenges that remain.

Send correspondence to B. T. Cox. bencox@mpb.ucl.ac.uk

Photons Plus Ultrasound: Imaging and Sensing 2009: The Tenth Conference on Biomedical Thermoacoustics, Optoacoustics, and Acousto-optics, edited by Alexander Oraevsky, Lihong V. Wang, Proc. of SPIE Vol. 7177, paper 717713 


\section{WHAT DOES A PHOTOACOUSTIC IMAGE ACTUALLY REPRESENT?}

As a first step in tackling the problem of quantitative photoacoustics it is worth reminding ourselves what a photoacoustic image is an image of. In other words, what is the source of contrast in a photoacoustic image? A photoacoustic image $i s$ related to the optical absorption in the tissue, as the incident photons must be absorbed to have any effect, but this relationship is rather less direct than is often assumed. A photoacoustic image is an estimate (typically to within a constant scaling factor) of the distribution of acoustic pressure that arises following the absorption of a pulse of light.

To see clearly what it is that a photoacoustic image depends on, we will briefly summarise the physics of photoacoustic signal generation. Consider a region of tissue that has an optical absorption coefficient distribution of $\mu_{a}(x)$. If the tissue is irradiated with a light pulse of duration $t_{p}$ then the distribution of optical energy, $h(x) \mathrm{Jm}^{-3}$, absorbed in the tissue during the pulse is

$$
h(x)=\int_{0}^{t_{p}} \mu_{a}(x) \Phi(x, t) d t=\mu_{a}(x) \phi(x)
$$

where $\Phi(x, t) \mathrm{Wm}^{-2}$ is the fluence rate describing the light distribution at a point $x$ in the tissue at time $t$, and $\phi \mathrm{Jm}^{-2}$ is its integral over time, often called simply the fluence. The fluence will depend on the optical properties of the medium, which in a highly scattering medium such as tissue can usually be characterised by the absorption and scattering coefficients, $\mu_{a}$ and $\mu_{s}$, and the anisotropy factor, $g$. As the incident fluence rate is of low power, in order to avoid tissue damage, and as the wavelength of the light is typically chosen to be in the near-infrared, in order to benefit from the optical 'window' at these wavelengths, the vast majority of the absorbed photons will be converted via vibrational relaxation to heat. The temperature rise, $\Delta T$, following the light pulse is related to the amount of 'deposited' heat by the specific heat capacity of the tissue. If it is assumed that the duration of the light pulse, $t_{p}$, is much shorter than the time is takes the density to change significantly, ie. the mechanical relaxation time of the tissue, then the relevant heat capacity is that for constant volume heating, $C_{v}$ :

$$
\Delta T=h / \rho_{0} C_{v}
$$

where $\rho_{0}$ is the mass density of the tissue. If the amount of heat energy deposited is small, it will cause small changes to the local density, pressure, $\Delta \rho, \Delta p$, as well as the temperature, in proportions given by the thermodynamic identity

$$
\Delta \rho=\rho_{0} K_{T} \Delta p-\rho_{0} \beta \Delta T
$$

where $K_{T}$ is the isothermal compressibility and $\beta$ is the volume thermal expansivity of the tissue. If, as above, it is assumed that the duration of the light pulse is much shorter than the time is takes the density to change significantly, ie. $\Delta \rho=0$, then Eq. (3) gives a relationship between the pressure and temperature changes in the tissue following the light pulse:

$$
\Delta p=\left(\beta / K_{T}\right) \Delta T
$$

Combining Eqs. (2) and (4) gives an expression for the increase in pressure as

$$
\Delta p=\left(\beta / K_{T} \rho_{0} C_{v}\right) h=\left(\beta c^{2} / C_{p}\right) h=\Gamma h
$$

where the first equality uses the substitution $c^{2}=C_{p} / K_{T} \rho_{0} C_{v}$ (with $C_{p}$ the specific heat capacity at constant presssure), and $\Gamma=\beta c^{2} / C_{p}$ is a dimensionless thermodynamic property of the tissue called the Grüneisen parameter. The small - and spatially varying - increase in pressure that we have so far called $\Delta p$ will initiate an acoustic pressure wave because tissue is elastic and inertial and so supports propagating acoustic waves. In this sense, $\Delta p$ can be considered to be an initial condition of an acoustic (or ultrasonic) radiation problem. If in general the acoustic pressure at a point $x$ and time $t$ is denoted by $p(x, t)$, then $p_{0}(x) \equiv p(x, 0)=\Delta p$. The ultrasonic waves that propagate out are called photoacoustic waves, and they can be described by the following initial value problem:

$$
\begin{gathered}
\mathcal{L}_{w} p(x, t)=0 \\
p_{0}(x)=\Gamma(x) h(x) \quad \partial p / \partial t(x, 0)=0
\end{gathered}
$$


The physical reason for the second initial condition is that the particle velocity of the acoustic waves is zero at $t=0$. The linear wave operator $\mathcal{L}_{w}$ has been used here instead of $\left(c^{2} \nabla^{2}-\partial^{2} / \partial t^{2}\right)$ so as not to restrict the model to a non-absorbing wave equation for acoustically homogeneous media, but to leave room to include wave equations that incorporate ultrasonic absorption or variations in sound speed and density within the tissue.

Now let us return to the question of what a photoacoustic image is an image of. In photoacoustic imaging, measurements of the acoustic pressure $p\left(x_{s}, t\right), x_{s} \in S$, are made over a measurement surface $S$, and $p_{0}$ is estimated using image reconstruction algorithms that are designed to solve the reverse problem of Eqs. (6) (in a temporal sense) and estimate the initial acoustic pressure distribution $p_{0}(x)$. It can be seen, from Eqs. (1) and (6), that this initial pressure distribution can be written as the product of three quantities, the tissue's Grüneisen parameter, its absorption coefficient, and the fluence distribution, all of which may vary with position $x$ :

$$
p_{0}(x)=\Gamma(x) h(x)=\Gamma(x) \mu_{a}(x) \phi\left(x ; \mu_{a}, \mu_{s}, g\right)
$$

In this paper, the tasks involved in quantitative photoacoustic imaging are divided into two inverse problems: an acoustic inverse problem (estimating the initial acoustic pressure distribution, $p_{0}$, accurately) and an optical inverse problem (recovering absorption coefficient or chromophore distributions). When the optical pulse is sufficiently short, these can be treated separately because of the very different time scales on which acoustic and optical propagation takes place. They will be discussed in turn below.

\section{THE ACOUSTIC INVERSE PROBLEM}

Before any progress can be made on the optical aspects of quantitative photoacoustic imaging, it is necessary that initial acoustic pressure distributions be estimated accurately and quantitatively. (Perhaps in some multiwavelength applications the quantitative requirement can be relaxed to a requirement that there is a calibration factor that is constant although unknown.) The two main subdivisions of the acoustic inversion, ultrasonic measurement and photoacoustic image reconstruction, ${ }^{3-5}$ each have a large body of literature which there is no room to review in any detail here. Suffice it to say that a great deal of progress has been made in this area in recent years, so much so that some people may consider all the important aspects of this problem to have been solved. However, there are several areas in which there is room for improvement.

- Tissue-realistic models The early work on photoacoustic image reconstruction assumed an acoustically homogeneous, non-absorbing medium. Despite these simplifications, several of these algorithms have found fruitful use in experimental studies and demonstrations of photoacoustic imaging. However, for many tissue types, the assumptions of uniform sound speed and density, and zero absorption, are inaccurate, and models incorporating more realistic tissue properties have begun to be studied. ${ }^{6-15}$

- Broadband detection Photoacoustic waves are by their nature broadband and therefore require broadband detectors in order to be able to capture them properly. For instance, for sub-100 $\mu$ m imaging, an upper limit of several tens of $\mathrm{MHz}$ may be required, but the initial pressure distribution may also contain spatial variations that correspond to relatively low frequencies (below $100 \mathrm{kHz}$ ). If the image is to be a quantitatively and spatially accurate representation of $p_{0}$, rather than just showing where there are edges, the low as well as the high frequencies must be captured. Broadband PVDF and interferometric sensor arrays are available, ${ }^{16,17}$ but despite this, more-or-less resonant piezo-electric ultrasound detectors, with limited frequency ranges, are still in common use.

- Sensor response Ideally, the frequency and angle-dependent response of the ultrasound sensor will be measured or modelled ${ }^{18}$ and deconvolved from the measurements. However, some sensor arrays have elements sufficiently small for them to be reasonably approximated as point sources.

- Calibration In order to be able to calculate the absorbed optical energy from the initial pressure distribution, the latter will need to be known in absolute units of pressure. There are two aspects to this: the sensor must be calibrated over the full frequency range of interest and the image reconstruction algorithm must be exact and retain the calibration. In some circumstances it may be that ratios of images are used, 
thereby removing the need for a calibration factor at all, or an unknown calibration factor may be found as part of the overall inversion, eg. if multiwavelength data constrains the solution sufficiently, but these considerations will be specific to certain cases.

- Limited view If the emitted photoacoustic waves are recorded everywhere on a measurement surface surrounding an object, there will be sufficient data to reconstruct an image of that object uniquely. However, for many targets it is not possible to surround them with sensors, and an estimate of the initial pressure distribution must be obtained using incomplete data. It would be interesting to know how different exact reconstruction algorithms, all of which give the same solution in the case of ideal data, respond when faced with incomplete data. Some authors have begun to look at ways to incorporate additional information, such as range conditions on the data, to improve the reconstructions. ${ }^{5,19-21}$

\section{THE OPTICAL INVERSE PROBLEM}

\subsection{Spatially-varying Grüneisen parameter}

The starting points of this section are the initial pressure distribution, $p_{0}$, which we will assume to have been faithfully recovered (see previous section) and the equation relating it to the absorbed optical energy density:

$$
p_{0}(x)=\Gamma(x) h(x)
$$

Recall that the Grüneisen parameter, $\Gamma(x)$, is a thermodynamic property of the tissue. If $\Gamma$ is known then it is a simple matter of division to recover the absorbed optical energy density $h(x)$ from the initial pressure distribution $p_{0}(x)$. Most attempts to do quantitative photoacoustics to date have assumed that $\Gamma$ is uniform throughout the tissue and is known (or perhaps is a constant that can be found through calibration). In fact, this will not necessarily be the case. A preliminary glance at estimates of sound speed, $c$, volume thermal expansivity, $\beta$, and specific heat capacity, $C_{p}$, in different tissue types ${ }^{22-25}$ (which allows the Grüneisen parameter to be estimated as $\Gamma=\beta c^{2} / C_{p}$ ) suggests that $\Gamma$ can vary considerably between different tissue types, such as fat $(\Gamma \approx 0.7$ to 0.9$)$ and blood $(\Gamma \approx 0.25)$. (It should not be surprising that $\Gamma$ varies between tissue types as it is well known that sound speeds and specific heat capacities vary between tissues.) If these estimates are at all accurate, this degree of variation in $\Gamma$ is a cause for concern for quantitative photoacoustics, for it is not possible - without some additional information - to make even the first step in the optical inversion: the conversion from initial pressure distribution to absorbed optical energy density. Currently, we suggest that a larger study of the variation of $\Gamma$ in different types of tissue is needed to answer this question satisfactorily. In the sections below, in order to make some progress, it is assumed that $\Gamma$ is known and $h(x)$ can be recovered.

\subsection{Why conventional linear spectroscopy with photoacoustic images doesn't work}

The fact that multiwavelength images of $h$ cannot be used in a direct spectroscopic inversion to estimate chromophore concentrations is well-known, but it bears repeating as it is crucial and often misunderstood. If there are $K$ chromophores with concentrations $c_{k}(x)$ and specific absorption spectra $\alpha_{k}(\lambda)$ contributing to the absorption coefficient at a wavelength $\lambda$, then the absorption coefficient can be written as

$$
\mu_{a}(x, \lambda)=\sum_{k=1}^{K} c_{k}(x) \alpha_{k}(\lambda)
$$

If $\mu_{a}(x, \lambda)$ is known for at least $K$ wavelengths then it is possible to calculate the chromophore concentrations $c_{k}(x)$ using a linear (matrix) inversion. As the absorbed energy density distributions can be written as $h=\mu_{a} \phi$, it is tempting to assume that the fluence $\phi$ is a constant and to substitute $h / \phi$ into Eq. (8) to get

$$
h(x, \lambda)=\phi(x, \lambda) \sum_{k=1}^{K} c_{k}(x) \alpha_{k}(\lambda)
$$

Unfortunately, this appealingly simple, linear, approach to recovering the chromophore concentrations $c_{k}$ from the absorbed energy images $h$ fails because the wavelength-dependent fluence is not known. ${ }^{1}$ There are two apects to this problem: 
Spectral corruption due to wavelength-dependent fluence The wavelength dependence of the fluence arises because, for any point not on the tissue surface, the light has had to travel through an absorbing medium to reach it. This colouring of the fluence by the surrounding medium is not mysterious; anyone who has looked through coloured glass will understand how an absorbing layer, with a certain absorption spectrum, will color the light going through it. The phenomenon here is the same, except, because of the scattering, the light does not travel in a straight line but rather is scattered all around the tissue sample. It is therefore carrying with it the 'colour' of all the regions of tissue it has travelled through on its contorted journey. The photoacoustic spectrum, $h(\lambda)$ at a point will depend both on the absorption spectrum at that point, $\mu_{a}(\lambda)$ (which is what we want to measure) and on the fluence spectrum there, $\phi(\lambda)$ (which depends on the surrounding absorption and scattering).

Nonlinear dependence of the fluence on $\mu_{a}$ If, somehow, the wavelength dependent fluence, $\phi(x, \lambda)$, were able to be measured, then Eq. (9) shows that it would be possible to use a linear inversion to obtain estimates of the chromophore concentration distributions, $c_{k}(x)$. However, if measurements of $\phi$ are not available (as is usually the case) one way to proceed is to use a model to estimate $\phi(x, \lambda)$. If $\phi$ just depended on the experimental setup (where the light sources are, for instance) then $\phi(x, \lambda)$ could be estimated and the linear inversion could again proceed. Unfortunately, there is a further complication which arises here because $\phi$ depends on $\mu_{a}$, ie. it depends on the chromophore concentrations which we were hoping to recover from the model. Eq. (9) should really be written as

$$
h(x, \lambda)=\phi\left(x, \lambda, c_{k}(x)\right) \sum_{k=1}^{K} c_{k}(x) \alpha_{k}(\lambda)
$$

This equation makes clear the nonlinear dependence of the absorbed energy density images $h$ on the chromophore concentrations $c_{k}$. This is a separate problem from the spectral corruption above, but it makes it more difficult to overcome. The optical inverse problem is a nonlinear inverse problem.

\subsection{Difference and quotient images}

In 'difference images', a small change in an image is made more visible by subtracting the original image. For instance, if the use of a contrast agent leads to a small change in an image, but the image is dominated by endogenous contrast, then a difference between the image before and after the contrast agent could reveal more clearly the location of the contrast agent. Clearly, such a method is useful for qualitative imaging, but is difference imaging any use in quantitative photoacoustic imaging? A typical approach goes as follows:

1. measure first image $h_{1}=\mu_{a} \phi_{1}$

2. add contrast agent $\mu_{a} \rightarrow \mu_{a}+\delta \mu_{a}$

(or perhaps change to a different wavelength where there is a strong absorption peak)

3. measure second image $h_{2}=\left(\mu_{a}+\delta \mu_{a}\right) \phi_{2}$

4. calculate the difference image $h_{2}-h_{1}=\mu_{a}\left(\phi_{2}-\phi_{1}\right)+\delta \mu_{a} \phi_{2} \approx \delta \mu_{a} \phi_{2}$

The equation in the last step above shows that even when the fluence is virtually unchanged, $\left(\phi_{2}-\phi_{1}\right) \approx 0$, * and it is therefore reasonable to neglect the first term, the difference image $\left(h_{2}-h_{1}\right)$ still depends on the unknown fluence $\phi_{2}$, and so is of no more use in the quantitative problem than the original images. ${ }^{26}$

Compare this with a quotient image, obtained by dividing the second image by the first:

$$
\frac{h_{2}}{h_{1}}=\frac{\left(\mu_{a}+\delta \mu_{a}\right) \phi_{2}}{\mu_{a} \phi_{1}} \approx 1+\frac{\delta \mu_{a}}{\mu_{a}}
$$

\footnotetext{
${ }^{*}$ In practice, in the case where the change in absorption coefficient arises through a change in wavelength to an absorption peak, it is unlikely that the fluence distribution will remain the same.
} 
Here, if $\left(\phi_{2}-\phi_{1}\right) \approx 0$, then the quotient image is proportional to the $\%$ change in the absorption coefficient and is no longer dependent on the fluence. A numerical example of this is given in Fig. 1. While this does not give the absolute absorption coefficient, it does give an image untainted by the fluence distribution, and in some circumstances knowing the $\%$ change in $\mu_{a}$ is all that is required. It should be noted that, in practice, quotient images may be rather sensitive to noise where $h_{1}$ takes small values.
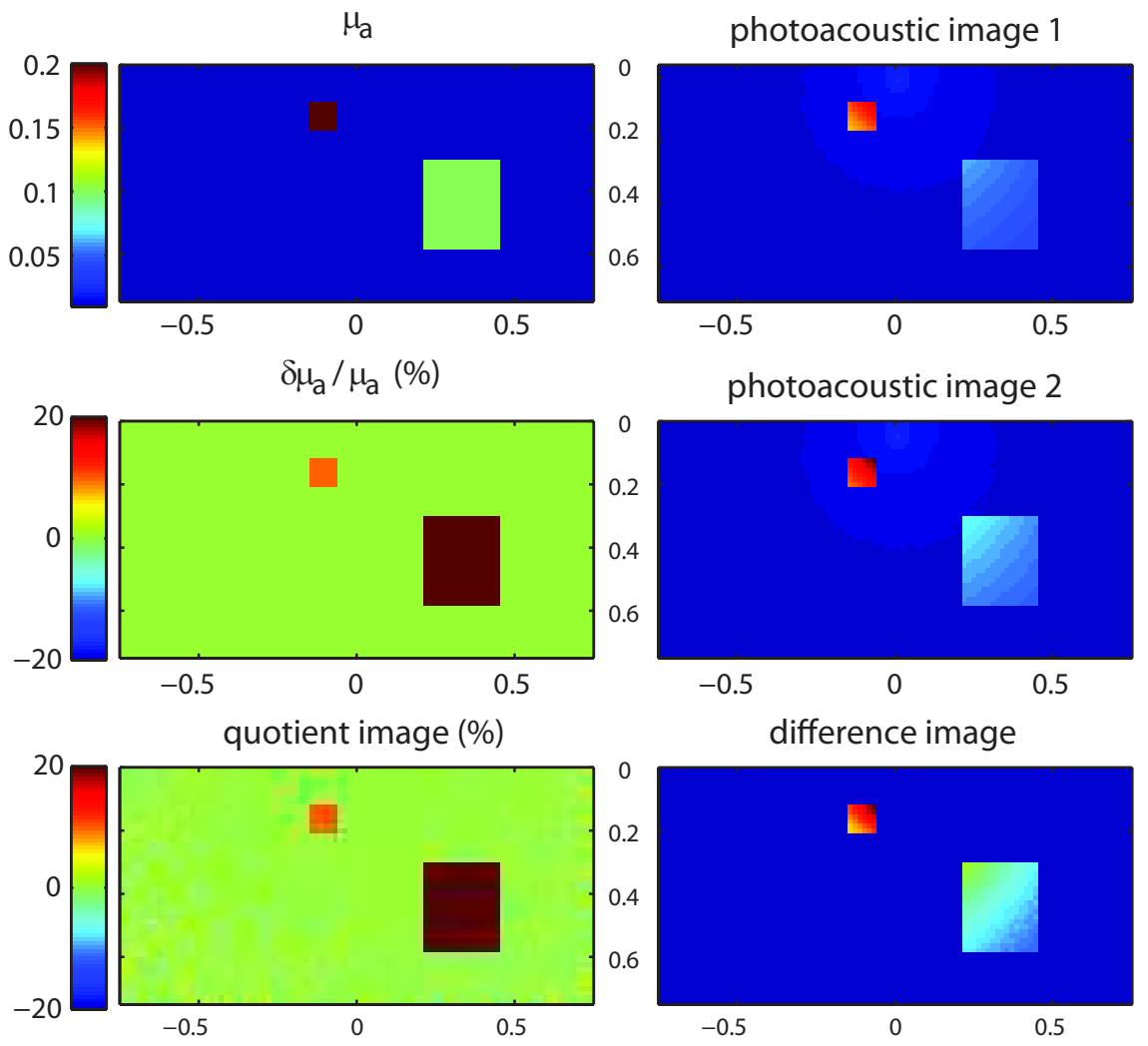

Figure 1. The absorption coefficient distribution, $\mu_{a}$ in $\mathrm{mm}^{-1}$, (top left) gives rise to 'photoacoustic image 1' (top right) when illuminated with a pencil beam at point $(0,0)$. When the absorption coefficient is perturbed by up to $20 \%$ (middle left) then 'photoacoustic image 2' results. The difference between these images (bottom right) still carries with it the effect of the non-uniform fluence. The quotient image (bottom left) recovers the $\%$ change in the absorption coefficient, but is more sensitive to noise.

\subsection{Model-based optical inversions}

Returning to the discussion in Section 4.2, despite the fact that $h$ is nonlinearly dependent on $\mu_{a}$, all hope is not lost, as $\mu_{a}$ can be recovered by using a numerical model to calculate $\phi$ (when the other optical coefficients, $\mu_{s}$ and $g$, are known) and by iterating from $\mu_{a}^{(0)}=0$ using

$$
\mu_{a}^{(n+1)}=\hat{h}(x) /\left(\phi\left(\mu_{a}^{(n)}\right)+\sigma\right)
$$

where $\sigma$ is a regularization term and $\hat{h}$ indicates measured data. ${ }^{27,28}$ Unfortunately, when $\mu_{s}$ and $g$ are not known, this iterative inversion is no longer suitable. A more general iterative scheme would be to invert for the absorption and scattering coefficients simultaneously (we assume in the discussion below that the anisotropy factor $g$ is known). Standard least-squares optimisation approaches could be used to minimise the functional

$$
\mathcal{E}=\int_{V}\left(\hat{h}-h\left(\mu_{a}, \mu_{s}\right)\right)^{2} d V
$$


but attempts to do this will soon run into the problem that a given $h$ is not uniquely associated to a single pair of coefficients $\left(\mu_{a}, \mu_{s}\right)$. In other words, it is possible to find two sets of coefficients $\left(\mu_{a 1}, \mu_{s 1}\right)$ and $\left(\mu_{a 2}, \mu_{s 2}\right)$ that result in the same absorbed energy image $h .{ }^{26}$ To overcome this non-uniqueness it is necessary to include some other information to restrict the space of solutions.

Recently, it has been shown that by using multi-wavelength data and an assumption about the wavelength dependence of the scattering coefficients it is possible to overcome this non-uniqueness and estimate chromophore concentration and scattering simultaneously. ${ }^{1,26}$ Another suggestion is that a set of images measured with the light source in different positions will give somewhat independent data sets and allow the absorption and scatter to be recovered simultaneously. ${ }^{29}$

\subsection{Large-scale inverse problem}

As computers and computing power becomes ever more readily available, it is tempting to think that the size of an inverse problem is not relevant to its successful solution once the basic problems of non-uniqueness and the existence of a solution have been overcome. However, some basic considerations show that the optical inverse problem discussed above can quickly become a very large scale problem: consider a 3D photoacoustic image of size $1 \mathrm{~cm}^{3}$ with $100 \mu \mathrm{m}$ resolution. Such an image has a million voxels. A optical inversion that uses a Newton or quasi-Newton inversion requires the storage of a hessian (second-order derivative) matrix. An inversion for four chromophore distributions and a scattering coefficient would require a hessian with $25 \times 10^{12}$ elements which would require hundreds of TB of memory for storage alone, let alone any matrix manipulation that might be required. ${ }^{26}$

This does not make the problem insoluble, but it does restrict the types of solution methods that could be used to solve it. Full Newton inversions, in which the hessian matrix is calculated, stored and inverted, are unlikely to be appropriate. However, quasi-Newton methods that estimate the (perhaps sparse?) hessian matrix from gradient calculations, or purely gradient-based techniques in which the second order derivatives are never estimated, might provide a way to tackle the problem effectively.

\section{DISCUSSION}

The sections above may give the impression that quantitative photoacoustic imaging has some way to go to reach its goal of accurate images of chromophore concentrations. While in the completely general case this is true, progress has been made by making certain restrictions and assumptions to reduce the size and complexity of the general case, ${ }^{30}$ including

- restricting the number of unknown parameters in the inversion by dividing the spatially varying optical coefficients into constant-value regions,

- including the wavelength dependence of the scattering coefficient, either measured or modelled using Mie theory, for example,

- including the Grüneisen parameter as a spatially-varying function, but linking it to the distributions of the chromophore concentrations so as not to increase the parameter space.

\section{SUMMARY}

This paper is a very brief survey of the challenges faced by quantitative imaging. The task is divided into two inverse problems: acoustic and optical. Our conclusions are that

- the acoustic problem is mostly well understood, but on-going work is providing useful improvements in image accuracy. 
- the complete optical problem - recovering accurate, high resolution, 3D images of chromophore concentration distributions from photoacoustic images measured at multiple wavelengths - is challenging because of the unknown, wavelength-dependent fluence and its dependence on the absorption coefficient, the photoacoustic absorption-scattering non-uniqueness, the sheer scale of the full inversion, and perhaps the difficulty in knowing the spatial-varying Grüneisen parameter.

- the most promising way forward seems to be with more focus on a particular application or situation. Rather than asking whether the general quantitative problem can be solved in the abstract, the application-specific approach is to study a particular application and bring prior knowledge of that situation to bear on the inversion, to help remove the non-uniqueness and reduce the solution space to a manageable size.

\section{ACKNOWLEDGMENTS}

The authors would like to thank Simon Arridge and Brad Treeby for useful discussions. This work was supported by the Engineering and Physical Sciences Research Council, UK.

\section{REFERENCES}

1. J. G. Laufer, C. Elwell, D. Delpy, and P. Beard, "Quantitative spatially resolved measurement of tissue chromophore concentrations using photoacoustic spectroscopy: application to the measurement of blood oxygenation and haemoglobin concentration," Phys. Med. Biol. 52, pp. 141-168, 2007.

2. L. Wang, "Prospects of photoacoustic tomography," Med. Phys. 35, pp. 5758 - 5767, 2008.

3. M. Xu and L. V. Wang, "Photoacoustic imaging in biomedicine," Rev. Sci. Inst. 77, p. 041101, 2006.

4. M. Agranovsky, P. Kuchment, and L. Kunyansky, "On reconstruction formulas and algorithms for thermoacoustic and photoacoustic tomography," Preprint, p. arXiv:0706.1303v1, 2007.

5. P. Kuchment and L. Kunyansky, "Mathematics of thermoacoustic tomography," European J. Appl. Math. 19, pp. 191-224, 2008.

6. M. H. Xu, Y. Xu, and L. H. V. Wang, "Time-domain reconstruction-algorithms and numerical simulations for thermoacoustic tomography in various geometries," IEEE Trans. Biomed. Eng. 50(9), pp. 1086-1099, 2003.

7. J. Zhang and M. A. Anastasio, "Reconstruction of speed-of-sound and electromagnetic absorption distributions in photoacoustic tomography," Proc. SPIE 6086, p. 608619, 2006.

8. X. Jin and L. Wang, "Thermoacoustic tomography with correction for acoustic speed variations," Phys. Med. Biol. 51, pp. $6437-6448,2006$.

9. M. Agranovsky and P. Kuchment, "Uniqueness of reconstruction and an inversion procedure for thermoacoustic and photoacoustic tomography with variable sound speed," Inverse Problems 23, pp. 2089-2102, 2007.

10. Y. Hristova, P. Kuchment, and L. Nguyen, "On reconstruction and time reversal in thermoacoustic tomography in acoustically homogeneous and inhomogeneous media," Inverse Problems 24, p. 055006, 2008.

11. P. L. Riviére, J. Zhang, and M. Anastasio, "Image reconstruction in optoacoustic tomography for dispersive acoustic media," Optics Letters 31, pp. 781-783, 2006.

12. P. Burgholzer, H. Grün, M. Haltmeier, R. Nuster, and G. Paltauf, "Compensation of acoustic attenuation for high-resolution photoacoustic imaging with line detectors," Proc. SPIE 6437, p. 643724, 2007.

13. B. E. Treeby and B. T. Cox, "Fast, tissue-realistic models of photoacoustic wave propagation for homogeneous attenuating media," Proc. SPIE 7177, p. in print, 2009.

14. D. Modgil, M. Anastasio, and P. L. Riviére, "Photoacoustic image reconstruction in an attenuating medium using singular-value decomposition," Proc. SPIE 7177, p. in print, 2009.

15. D. Modgil, M. Anastasio, K. Wang, and P. L. Riviére, "Image reconstruction in photoacoustic tomography with variable speed of sound using a higher-order geometrical acoustics approximation," Proc. SPIE $\mathbf{7 1 7 7}$, p. in print, 2009. 
16. L. Brown, "Design considerations for piezoelectric polymer ultrasound transducers," IEEE Trans. UFFC 47, pp. 1377-1396, 2000.

17. E. Z. Zhang, J. G. Laufer, and P. C. Beard, "Backward-mode multiwavelength photoacoustic scanner using a planar Fabry-Perot polymer film ultrasound sensor for high-resolution three-dimensional imaging of biological tissues," Applied Optics 47, pp. 561-577, 2008.

18. B. T. Cox and P. C. Beard, "The frequency-dependent directivity of a planar Fabry-Perot polymer film ultrasound sensor," IEEE Trans. UFFC 54, pp. 394-404, 2007.

19. Y. Xu, L. V. Wang, G. Ambartsoumian, and P. Kuchment, "Reconstructions in limited-view thermoacoustic tomography," Med. Phys. 31, pp. 724-733, 2004.

20. S. K. Patch, "Thermoacoustic tomography - consistency conditions and the partial scan problem," Phys. Med. Biol. 49, pp. 2305-2315, 2004.

21. M. A. Anastasio and J. Zhang, "Image reconstruction in photoacoustic tomography with truncated cylindrical measurement apertures," Proc. SPIE 6086, p. 608610, 2006.

22. F. Duck, Physical Properties of Tissue: A Comprehensive Reference Book, Academic Press, London, 1990.

23. A. S. T. Blake, G. W. Petley, and C. D. Deakin, "Effects of changes in packed cell volume on the specific heat capacity of blood: implications for studies measuring heat exchange in extracorporeal circuits," British Journal of Anaesthesia 84, pp. 28-32, 2000.

24. T. Azuma, K. Sasaki, K. i. Kawabata, and S. i. Umemura, "Tissue expansion imaging for tissue coagulation mapping during high intensity focused ultrasound therapy," IEEE Ultrasonic Symposium , pp. 1770-1773, 2006.

25. M. Toubal, M. Asmani, E. Radziszewski, and B. Nongaillard, "Acoustic measurement of compressibility and thermal expansion coefficient of erythrocytes," Phys.Med. Biol. 44, pp. 1277-1287, 1999.

26. B. T. Cox, S. R. Arridge, and P. C. Beard, "Estimating chromophore distributions from multiwavelength photoacoustic images," J. Opt. Soc. Am. A 26, pp. 443-455, 2009.

27. B. T. Cox, S. R. Arridge, K. Köstli, and P. C. Beard, "2D quantitative photoacoustic image reconstruction of absorption distributions in scattering media using a simple iterative method," Appl. Opt. 45(8), pp. 1866-1874, 2006.

28. B. Banerjee, S. Bagchi, R. M. Vasu, and D. Roy, "Quantitative photoacoustic tomography from boundary pressure measurements: noniterative recovery of optical absorption coefficient from the reconstructed absorbed energy map," J. Opt. Soc. Am 25, pp. 2347-2356, 2008.

29. X. Chen, K. Mathewson, and R. Zemp, "Laminar photoacoustic microscopy for optical scattering measurements," SPIE, Photons Plus Ultrasound, San Jose 2009.

30. J. G. Laufer, B. T. Cox, E. Z. Zhang, and P. C. Beard, "Quantitative determination of chromophore concentrations from photoacoustic images using a model-based inversion technique," Applied Optics, to be submitted , 2009. 